\title{
The Analysis of Instantaneous Polarization Attribute in Crosswell Seismic Wave Field
}

\author{
Dai Yu *, Yuan Sun, Dong Li, Hui Wang \\ College of Geology Engineering and Geomatics \\ Chang'an University \\ Xi'an, China \\ tablemax47@126.com
}

\author{
Guangyi Hu \\ Development Institute of CNOOC Research Institute \\ CNOOC \\ Beijing, China
}

\begin{abstract}
In the stage of oilfield development, the fractures of clasolite reservoir begin to develop after the process of fracturing and water flooding. At the same time, the lateral heterogeneity has the significant changes, and this situation will cause the abnormal changes of many seismic attribute parameters. Based on theoretical analysis of polarization attributes of threecomponent borehole seismic wave field, which attributes include the long axis, short axis, azimuth and polarization ratio, we can get the conclusion that it is possible to have some abnormal changes for those attributes. In this paper, we use the crosswell three-component wave field which is simulated by the anisotropic medium wave equation forward modeling, to calculate the instantaneous polarization attribute of the pre-stack seismic data, and implement the imaging process based on the method of wave field imaging. It turn out that there are some larger differences in polarization attribute between the horizontally layered model and fault model. It is obvious to see the characteristic of layered anisotropy. In the fault model, the fault point abnormity of the attribute in polarization ratio and ellipsoid ratio has the significant features. And this is used as the identification symbol of the fracture development zone.
\end{abstract}

Keywords_polarization ratio; ellipsoid ratio; attributes imaging; anisotropy ; fault

\section{INTRODUCTION}

In the stage of oilfield development, we often improve the recovery ratio in the manner of fracturing and water flooding. It is very important for us to know the fracture development zone and heterogeneity of reservoir in the process of injectionproduction development. In order to evaluate the effect of injection-production project, we usually use the method of one well monitoring, but using this method cannot get the prospective results in the aspect of changes of crosswell reservoir. For such problems, micro-seismic monitoring technology and borehole seismic monitoring technology are the effective ways to solve it. The former is to confirm the location of micro-seismic source by arrival time difference of microseismic wave, and the micro-seismic wave generated by fracturing and water flooding received by different monitoring stations. In this way, we can calculate the spread range of fracturing and water flooding ${ }^{[1][6]}$. The latter is to extract the polarization attribute of seismic wave field by using the threecomponent data collected by borehole seismic. At the same time, we can confirm the range of fracture development ${ }^{[4][5]}$ and forecast the location of waterflood front by analyzing abnormal attributes. In practice, the combination of multiple attribute can forecast the fractures of clasolite reservoir and the change features of oil-water relation ${ }^{[2][3]}$.

As for as the crosswell seismic, the three-component wave field information of pre-stack common-shot-point or commonreceiver-point are of abundance. But it cannot intuitively express abnormal characteristics of crosswell reservoir. In this paper, we take advantage of the fundamental method of crosswell seismic wave field imaging, to use the crosswell three-component wave field which is simulated by the VTI medium wave equation forward modeling, to extract the instantaneous polarization attribute of the pre-stack seismic data, and implement the imaging process. It turn out that there are some larger differences in polarization attribute between the horizontally layered model and fault model. It is obvious to see the characteristic of layered anisotropy. In the fault model, the fault point abnormity of the attribute in polarization ratio and ellipsoid ratio has the significant features. And this is used as the identification symbol of the fracture development zone.

\section{Method And Technology}

\section{A. Introduction of Polarization Attribute}

In essence, seismic wave filed is the outcome of vibration which has different types and polarization attributes and acquired by mutual intervening and stacking. And the single seismic wave is linear and has different polarization directions. For instance, the direction of propagation of p-wave is consistent with the particle vibrating direction. According to the analysis of polarization in seismic data, we can get the spatial polarization characteristics and characteristic parameter of seismic wave field. The characteristics include the long axis, short axis, secondary short axis, polarization ratio, incident angle and azimuth, etc. And long axis, short axis, secondary short axis, ellipsoid ratio (the ratio of long and short axis) as well as polarization ratio indicate the movement path of the particle in three-dimensional space. Polarization ratio is also called polarization coefficient, its theoretical value between 0 and 1 . When the value gets 1 , it is linear polarization, and when it gets 0 , the polarization trace approximate to ellipsoid. It indicates that its abnormal change has the certain relativity with the reservoir anisotropy and fluid variation. Ellipsoid ratio 
indicates that the change of movement path of particle has the certain relativity with the reservoir heterogeneity. Through analyzing, we can know that the anomalous change of the two polarization attribute parameters are helpful for identifying minor fault and fracture development zone.

\section{B. Extraction and Imaging of Instantaneous Polarization Attribute}

1) Attribute Extraction ${ }^{[4][5]}$

Polarization attribute extraction in crosswell seismic is based on the crosswell three-component wave field. In general, there are many methods about extracting polarization attribute parameter described below. (1) Solved by the amplitude of wave arrival of $\mathrm{x}$ and $\mathrm{y}$ component. (2)Hodograph method. (3) Analytical method of energy criterion. (4)Covariance matrix method. (5) Radial energy method. As for the extraction of instantaneous polarization attribute parameters, using covariance matrix method is more suitable and its principle as follows:

Selecting $N$ sampling point time window $\left(T_{1}, T_{2}\right)$ in threecomponent record, in every sampling point $\mathrm{i}, X_{i} 、 Y_{i} 、 Z_{i}$ is the value of three-component and their mean value in time window $\left(T_{1}, T_{2}\right)$ as follows:

$$
m_{x}=\frac{1}{N} \sum_{i=N_{1}}^{N_{2}} x_{i}, \quad m_{y}=\frac{1}{N} \sum_{i=N_{1}}^{N_{2}} y_{i}, \quad m_{z}=\frac{1}{N} \sum_{i=N_{1}}^{N_{2}} z_{i}
$$

Among them, $\left(N_{2}-N_{1}\right) \Delta t=T_{2}-T_{1}$, and $\Delta t$ is sampling rate; $N=N_{2}-N_{1}+1$.

By applying the method of statistics to all the data of sampling point in time window, we can acquire the mean value of every component in time window and then obtain a covariance matrix:

$$
M_{c}=\frac{1}{N}\left[\begin{array}{ccc}
\sum A^{2} & \sum A B & \sum A C \\
\sum B A & \sum B^{2} & \sum B C \\
\sum C A & \sum C B & \sum C^{2}
\end{array}\right]
$$

$$
\text { Where } \sum \text { is } \sum_{i=N_{1}}^{N_{2}}, A=\left(x_{i}-m_{x}\right) ; B=\left(y_{i}-m_{y}\right) \text {; }
$$

$C=\left(z_{i}-m_{z}\right)$

By calculating the eigenvalue $\lambda_{1}, \lambda_{2}, \lambda_{3}$ of covariance matrix and its eigenvector, we then can obtain the parameters like long axis, short axis, secondary short axis and polarization ratio of particle vibration trace, as follows:

$$
a=\sqrt{3 \lambda_{1}}, b=\sqrt{3 \lambda_{2}}, c=\sqrt{3 \lambda_{3}}
$$

$$
R=\frac{\left(1-\frac{\lambda_{2}}{\lambda_{1}}\right)^{2}+\left(1-\frac{\lambda_{3}}{\lambda_{1}}\right)^{2}+\left(\frac{\lambda_{2}}{\lambda_{1}}-\frac{\lambda_{3}}{\lambda_{1}}\right)^{2}}{2\left(1+\frac{\lambda_{2}}{\lambda_{1}}+\frac{\lambda_{3}}{\lambda_{1}}\right)^{2}}
$$

The eigenvector, which belong to the eigenvalue, obtained by covariance matrix can determine the direction of every axis. And the biggest eigenvalue is $\lambda_{1}$ and its eigenvector is principal eigenvector $V\left(V_{x}, V_{y}, V_{z}\right)$. The energy of signal mostly focus on the $\lambda_{1}$. In $x y z$ coordinates system, the principal eigenvector $V$ can be only obtained by three scalar coordinates $\left(V_{x}, V_{y}, V_{z}\right)$ and the direction of $V$ is determined by its three direction cosine $\cos \alpha(t), \cos \beta(t), \cos \gamma(t) . \alpha(t), \beta(t), \gamma(t)$ is the angle between the principal eigenvector and three coordinates axis $x, y, z$. By applying the space geometry relation and $V_{x}^{2}+V_{y}^{2}+V_{z}^{2}=1$, we can draw a conclusion that the three direction cosine of principal eigenvector $V$ is just its scalar coordinates and illustrated as follows:

$$
\left\{\begin{array}{l}
\cos \alpha(t)=V_{x} \\
\cos \beta(t)=V_{y} \\
\cos \gamma(t)=V_{z}
\end{array}\right.
$$

Azimuth $\theta$ and incident angle $\varphi$ of principal signal polarization can be calculated by the direction cosine.

$$
\left\{\begin{array}{l}
\theta=\arctan (\cos \beta / \cos \alpha) \\
\varphi=\operatorname{arc}(\cos \gamma)
\end{array}\right.
$$

Based on these formulas, we can obtain the polarization parameters which belong to the central location in time window. By sliding the time window point by point, we can obtain all the polarization parameters of wave field in one trace.

\section{2) Attribute Imaging}

The pre-stack polarization attribute parameter can only show the information about shot gather or trace gather, and we have to do the process of imaging in the case of interpretation. In this paper, we extract CLP gathers and then do normal move out correction and stack in crosswell seismic reflection wave field. The method above has three steps. (1)scanning the stack velocity on the basis of crosswell seismic reflection wave field. (2) computing the stack velocity so as to do normal move out correction to the polarization record of common shot gathers in crosswell seismic which including vertical and horizontal normal move out correction. At the same time, we extract the CLP gather of polarization attribute. (3) In the methods of stack imaging, we can use conventional stack method and median stack method. Median stack method has better fidelity so that we can acquire the imaging section of crosswell seismic polarization attribute. (See Fig. 6 and 7.) 


\section{ANALYSIS OF THEORY MODEL}

In order to test the features that polarization attributes with regard to inhomogeneity of stratum and fault, we devised two kinds of geologic model respectively, which is crosswell horizontal layered anisotropy (VTI medium) model and fault model. The basic parameters of model are illustrated in figure 1 and table I . By using VTI medium wave equation, the threecomponent wave field in crosswell can be simulated from forward modeling and the parameters of observation system are listed in TABLE II. The component of $\mathrm{p}$ and $\mathrm{s}$ wave after the process of three-component composition are described in figure 2 and 3. By comparing them, we can realize that there are some larger differences in first break of $\mathrm{p}$ and $\mathrm{s}$ wave and up-going reflection wave field which synthesized by horizontal layered model and fault model.

TABLE I. HORIZONTAL LAYERED VTI MEDIUM MODEL AND FAUlT MODEL

\begin{tabular}{|c|c|c|c|c|c|c|c|c|}
\hline \multirow{2}{*}{$\begin{array}{c}\text { Type } \\
\text { of } \\
\text { Med } \\
\text { ium }\end{array}$} & \multicolumn{2}{|c|}{$\begin{array}{c}\text { Depth Range } \\
(\mathrm{m})\end{array}$} & \multirow{2}{*}{$\begin{array}{c}\text { Dens } \\
\text { ity } \\
(\mathrm{g} / \mathrm{c} \\
\left.\mathrm{m}^{3}\right)\end{array}$} & \multirow[b]{2}{*}{$C_{11}$} & \multirow[b]{2}{*}{$C_{13}$} & \multirow[b]{2}{*}{$C_{33}$} & \multirow[b]{2}{*}{$C_{44}$} & \multirow[b]{2}{*}{$C_{66}$} \\
\hline & $\begin{array}{c}\text { Model } \\
1\end{array}$ & $\begin{array}{c}\text { Model } \\
2 \\
\text { (fault } \\
\text { ) }\end{array}$ & & & & & & \\
\hline \multirow{5}{*}{ VTI } & $\begin{array}{l}0 \sim \\
200\end{array}$ & $\begin{array}{l}0 \sim \\
260\end{array}$ & 2.20 & 3.254 & 1.278 & 1.664 & 0.467 & 1.281 \\
\hline & $\begin{array}{c}200 \sim \\
300\end{array}$ & $\begin{array}{c}260 \sim \\
350\end{array}$ & 2.32 & 5.246 & 2.047 & 2.682 & 0.753 & 2.065 \\
\hline & $\begin{array}{c}300 \sim \\
400\end{array}$ & $\begin{array}{l}350 \sim \\
470\end{array}$ & 2.25 & 3.091 & 1.206 & 1.580 & 0.444 & 1.217 \\
\hline & $\begin{array}{c}400 \sim \\
600\end{array}$ & $\begin{array}{c}470 \sim \\
660\end{array}$ & 2.34 & 1.040 & 0.270 & 1.040 & 0.192 & 0.192 \\
\hline & $\begin{array}{c}600 \sim \\
800\end{array}$ & $\begin{array}{c}660 \sim \\
800\end{array}$ & 2.50 & 2.860 & 1.212 & 2.250 & 0.732 & 0.732 \\
\hline
\end{tabular}

TABLE II. OBSERVATION SYSTEM PARAMETERS

\begin{tabular}{|c|c|c|c|}
\hline $\begin{array}{c}\text { Model Size } \\
(\mathbf{m})\end{array}$ & $800 \times 800 \times 800$ & $\begin{array}{c}\text { Wellhead } \\
\text { Coordinates }\end{array}$ & $\begin{array}{c}(200,200,0) \\
(600,600,0)\end{array}$ \\
\hline $\begin{array}{c}\text { Number of } \\
\text { Model } \\
\text { Layers }\end{array}$ & 5 & $\begin{array}{c}\text { Number of } \\
\text { Channels }\end{array}$ & $\begin{array}{c}81 \text { (increase from } \\
\text { the shallow to } \\
\text { deep })\end{array}$ \\
\hline $\begin{array}{c}\text { Grid } \\
\text { Spacing }\end{array}$ & $10 \mathrm{~m}$ & $\begin{array}{c}\text { Trace } \\
\text { Intervals }\end{array}$ & $10 \mathrm{~m}$ \\
\hline $\begin{array}{c}\text { Well } \\
\text { Pattern }\end{array}$ & vertical well & Sampling Rate & $500 \mathrm{us}$ \\
\hline $\begin{array}{c}\text { Shot } \\
\text { Position }\end{array}$ & $(200,200,400)$ & $\begin{array}{c}\text { Record } \\
\text { Length }\end{array}$ & $1.5 \mathrm{~s}$ \\
\hline
\end{tabular}

Fig. 1. Anisotropy Horizontally Layered Geologic Model (left) and Fault
Geologic Model (right).
According to the $\mathrm{P}, \mathrm{SV}$ and $\mathrm{SH}$ component which by synthesized from three-component, we can extract the polarization attributes shot gather record of forward modeling on the method of covariance matrix in the two models above. At the same time, we can obtain the polarization ratio and ellipsoid ratio. (See in figure 4 and 5). We also can see that there are some differences about the shot gather polarization attributes in faulted and unfaulted model. In order to analyze and interpret easily, we do the imaging process to polarization ratio and ellipsoid ratio and the result of process is shown in figure 6 and 7. Through analyzing the imaging result of polarization attributes of unilateral shot, we can know that the lateral discontinuous changes of polarization attributes in section and abnormal change in vertical and horizontal direction have direct relation with fault and layered anisotropy of medium. By comparing the imaging section of faulted and unfaulted model, we can draw the conclusion that the fault point in polarization attribute has obvious abnormal characteristics and the spatial position in accordance with the designed model.

\section{CONCLUSION}

Through testing the theoretical model we can find the reasons of abnormal change in polarization attributes as follows: (1)Fault and the fracture development, abruptly change of stratum in horizontal direction, including offset, wedge out and asymmetrical change in thickness, etc. It often leads to abruptly change of polarization attributes. (2)Spatial change of elastic property in reservoir (such as transversely isotropic media VTI), it can lead to the layered abnormal change of polarization attributes. When we consider the information of fault, the degree of change will become more complicated. The testing results show that when we conduct suitable imaging process on the instantaneous polarization attributes in crosswell seismic record extracted by covariance matrix, we can obtain the feasible results. Although we only used crosswell seismic one unilateral shot three-component wave field which has incomplete imaging section, it turn out that this method is effective. The result we get can help us to identify the inhomogeneous changes of crosswell reservoir as well as the fault, the fracture development zone, etc.
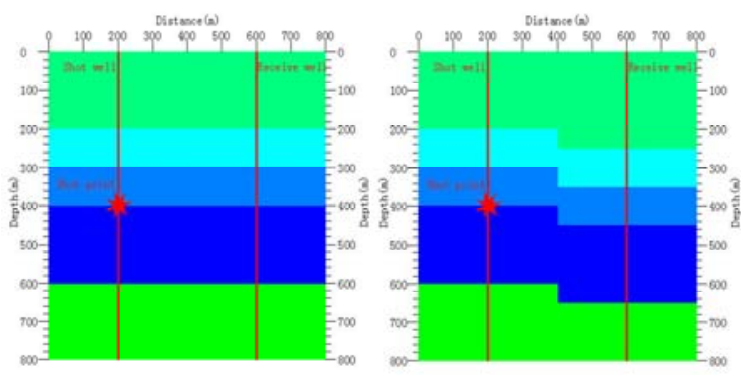


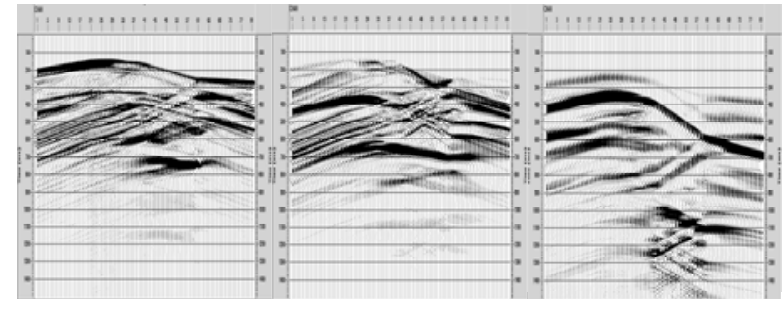

Fig. 2. Time-space Domain Wave Field of P(left), SV(middle)and SH(right)Component which Synthesized by Three-component in Horizontal Layered Model.

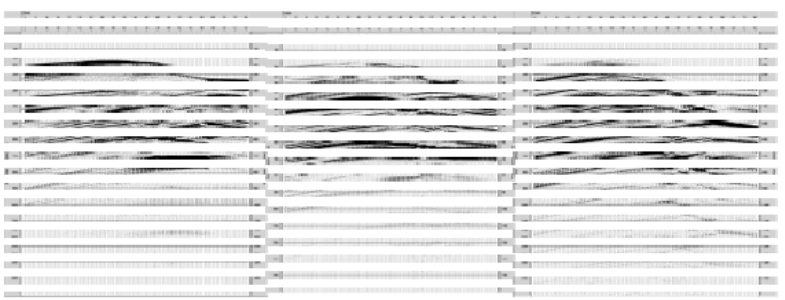

Fig. 3. Time-space Domain Wave Field of P(left), SV(middle)and SH(right)Component which Synthesized by Three-component in Fault Model.

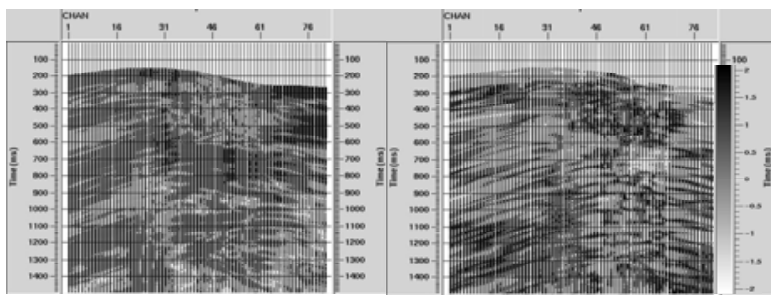

Fig. 4. Single-shot Record in Horizontal Layered Model about the Attributes of Polarization Ratio (left) and Ellipsoid Ratio (right)

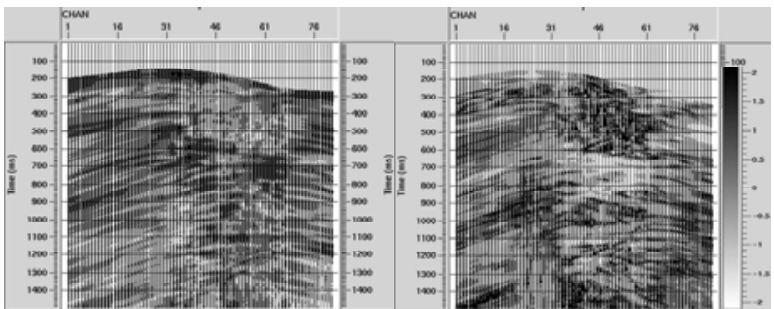

Fig. 5. Single-shot Record in Fault Model about the Attributes of Polarization Ratio (left) and Ellipsoid Ratio (right)

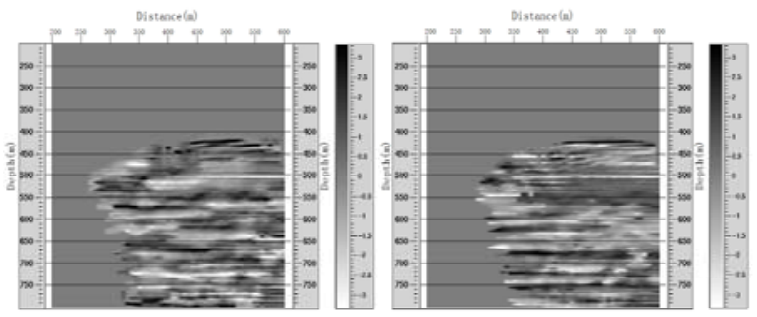

Fig. 6. Imaging Depth Section in Horizontal Layered Model about the Attributes of Polarization Ratio (left) and Ellipsoid Ratio (right)
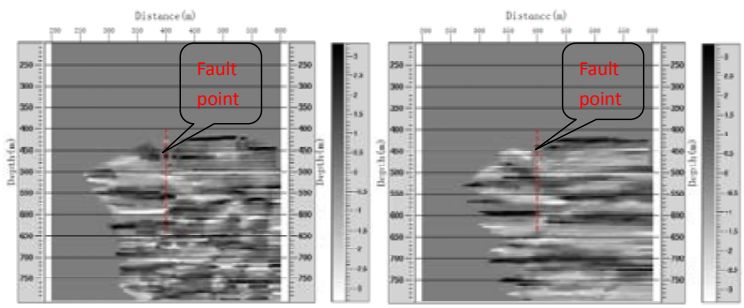

Fig. 7. Imaging Depth Section in Fault Model about the Attributes of Polarization Ratio (left) and Ellipsoid Ratio (right)

\section{ACKNOWLEDGMENT}

This research work was partially supported by China National Major Science and Technology Project (subproject No: 2011ZX05024-001-03).

\section{REFERENCES}

[1] Zhang Chunyan, Zhu Lilin, Jin Baozhen, Wu Jun, and Li Nengwu, "The Evaluation on Improving Waterflooding Effect of Micro-seismic Monitoring Technology” [J]. Oil-GasField Surface Engineering, 2010, 29(11), pp. 35-36.

[2] Gu Wen, "Methods and Technology Research on Seismic Multi-attribute Fracture Detection" [D]. Chengdu University of Technology, 2012.

[3] Xie Zhan'an, and Zhao Yan, "Application and Effect of Seismic Attribute Analysis on G29 Block"[J]. Journal of Oil and Gas, 2005, 27(4), pp. 444-446.

[4] Li Xiaowei, "Application Research and Parameter Extraction of Inclined Well Crosswell Seismic Attribute of P and S Wave”[D]. Chang'an University, 2013.

[5] Liu Ye, "The Research on the Method of VSP Three-component Instantaneous Polarization”[D]. Chang'an University, 2009.

[6] Du Juan, and Yang Shumin, "The Analysis on Field Application Results of Micro-seismic Monitoring Technology in Crosswell”[J]. Daqing Petroleum Geology and Development, 2007, 26(4), pp. 120-122. 